\title{
Human epidermal growth factor receptor 2-, epidermal growth factor receptor-, and mesenchymal epithelial transition factor-positive sites of gastric cancer using surgical samples
}

\author{
Yasuhiro Oono ${ }^{1}$ (D) Takeshi Kuwata $^{2} \cdot$ Kenji Takashima $^{1} \cdot$ Kensuke Shinmura $^{1} \cdot$ Keisuke Hori $^{1} \cdot$ Yusuke Yoda $^{1}$. \\ Hiroaki Ikematsu ${ }^{1} \cdot$ Kohei Shitara $^{3} \cdot$ Takahiro Kinoshita $^{4} \cdot$ Tomonori Yano $^{1}$
}

Received: 5 April 2018 / Accepted: 24 June 2018 / Published online: 27 June 2018

(c) The International Gastric Cancer Association and The Japanese Gastric Cancer Association 2018

\begin{abstract}
Background Receptor tyrosine kinases (RTKs) play critical roles in gastric cancer (GC) progression and are potential targets for novel molecular-targeted agents or photo-immunotherapies. During patient selection, targeted biopsy is the first step. However, heterogeneous expression of RTKs based on the macroscopic appearance in GC has not been extensively addressed. Accordingly, in this study, we evaluated differences in RTK expression associated with macroscopic appearance in GC.

Methods In total, 375 consecutive patients who had undergone gastrectomy at the National Cancer Center Hospital East and who had histologically proven adenocarcinoma, available archived tumor sample, and no history of chemotherapy were enrolled in this study. For these cases, tissue microarray (TMA) samples were examined using immunohistochemistry (IHC). Based on the results of IHC, cases were selected for detailed examination. We re-evaluated IHC scores in more than three tumor blocks per case and comparatively evaluated differences in IHC expression in RTKs between the mucosal portion (MuP) and invasive portion (InP).

Results Human epidermal growth factor receptor 2 (HER2)-, epidermal growth factor receptor (EGFR)-, and mesenchymal epithelial transition factor (c-MET)-positive rates were 6,9 , and 20\%, respectively. Twenty-two cases were then analyzed to assess differences in IHC expression levels in the same lesion. Concordance rates of positive staining of HER2, EGFR, and MET between MuP and whole tumor were 100, 40, and 56\% and those with InP were 46, 100, and 56\%.

Conclusions To avoid underestimating expression status, biopsies must be taken from MuP for HER2, InP for EGFR, and both proportions for c-MET.
\end{abstract}

Keywords Human epidermal growth factor receptor 2 - Epidermal growth factor receptor · Mesenchymal epithelial transition factor $\cdot$ Gastric cancer

Yasuhiro Oono

yohno@east.ncc.go.jp

1 Department of Gastroenterology and Endoscopy, National Cancer Center Hospital East, 6-5-1 Kashiwanoha, Kashiwa, Chiba 277-8577, Japan

2 Department of Pathology and Clinical Laboratories, National Cancer Center Hospital East, Kashiwa, Japan

3 Department of Gastroenterology and Gastrointestinal Oncology, National Cancer Center Hospital East, Kashiwa, Japan

4 Gastric Surgery Division, National Cancer Center Hospital East, Kashiwa, Japan

\section{Background}

In 2015, gastric cancer (GC) was the fifth most common malignancy $(1,313,000$ cases, $7.5 \%$ of all cancers) and the third leading cause of cancer-related death worldwide (819,000 deaths, $9.4 \%$ of all cancer-related deaths) [1]. Although the most effective treatment for localized disease is surgery, more than $60 \%$ of patients who undergo curative resection ultimately experience recurrence [2-4]. The prognosis of patients with advanced or recurrent GC remains poor. Therefore, the development of novel molecular-targeted agents for treating advanced or recurrent GC is urgently required.

Some receptor tyrosine kinases (RTKs) play critical roles in GC progression and are potential targets for novel 
molecular-targeted agents [5-7]. Human epidermal growth factor 2 (HER2) was introduced as a predictive biomarker for the treatment of GC with trastuzumab [8]. Trastuzumab, a recombinant monoclonal antibody targeting the HER2 protein, has recently been approved in many countries for the treatment of metastatic adenocarcinomas of the stomach and gastroesophageal junction (GEJ) [9, 10]. Although many studies have previously evaluated HER2 status in GC, the patient cohorts and scoring criteria have varied, resulting in discrepancies in rates of HER2-positive cancer, varying from 4 to $53 \%$, with a median rate of $18 \%$ [11]. Similarly, the prognostic impact and clinicopathological features of epidermal growth factor receptor (EGFR) and mesenchymal epithelial transition factor (c-MET) have also been studied. EGFR overexpression, which has been observed in 27-44\% of patients with GC, is generally found to be a poor prognostic factor [12-15]. Similarly, c-MET overexpression, which has been observed in $22-82 \%$ of patients with GC, has also been reported to be associated with poor prognosis [16-21].

During patient selection for molecular-targeted agents or photoimmunotherapy (PIT), targeted biopsy under endoscopic observation is the first step. However, RTK expression in GC is highly heterogeneous [22-28]. Only small biopsy samples are available in most patients with unresectable or metastatic disease, and intratumor heterogeneity may be a potential cause for the failure of targeted therapy in GC [29]. However, heterogeneous expression of RTKs, as determined by analysis of macroscopic appearance in GC, has not been extensively addressed. Therefore, to avoid sampling error, it is important to take samples from an appropriate tumor site endoscopically.

Accordingly, in this study, we investigated the heterogeneity of RTKs, including HER2, EGFR, and c-MET, based on the macroscopic appearance of GC.

\section{Materials and methods}

\section{Cases and tissue microarray (TMA)}

TMA construction was described previously by Aizawa et al. [30]. Briefly, formalin-fixed paraffin-embedded specimens from 414 consecutive patients who had undergone gastrectomy at our hospital between August 2011 and March 2015 were selected to construct the TMAs. For each clinical case, representative tumor areas were selected, and tissue cores (each $2.0 \mathrm{~mm}$ in diameter) were assembled in a TMA format. Serial $4-\mu \mathrm{m}$-thick sections were prepared and used for hematoxylin and eosin staining and immunohistochemistry (IHC).

\section{Patients and data collection}

The criteria for inclusion were as follows: (1) histologically proven gastric or GEJ (type I-III tumors using the Siewert classification; [31]) adenocarcinoma, (2) available archived tumor sample, and (3) no history of chemotherapy. Exclusion criteria included patients' refusal of permission for the use of clinical data and tumor tissue samples.

We retrospectively collected the following clinicopathological data: sex, age, primary tumor location, histological classification (Lauren classification), Borrmann classification, and T stage (AJCC 7th edition).

\section{HER2, EGFR, and C-MET IHC}

Formalin-fixed paraffin-embedded tumor samples were examined for HER2, EGFR, and c-MET using IHC. HER2 IHC analysis was performed using the PATHWAY antiHER-2/neu (4B5) rabbit monoclonal primary antibody (Ventana Medical Systems, Tucson, AZ, USA). The intensity of membrane staining was evaluated according to the HER2 scoring system, which indicated the need for treatment with trastuzumab by a subset analysis of the trastuzumab for GC (ToGA) trial [8]. Surgical specimen staining patterns were scored as follows: 0 , no reactivity or membranous reactivity in less than $10 \%$ of tumor cells; $1+$, faint/barely perceptible membranous reactivity in $10 \%$ or more of tumor cells or reactive only in part of their membrane; $2+$, weak to moderate complete or basolateral membranous reactivity in $10 \%$ or more of tumor cells; and $3+$, moderate to strong complete or basolateral membranous reactivity in $10 \%$ or more of tumor cells.

IHC for EGFR and c-MET was performed automatically using a Ventana BenchMark ULTRA with the CONFIRM anti-EGFR (3C6) primary antibody (Ventana Medical Systems) and the CONFIRM anti-total c-MET (SP44) rabbit monoclonal primary antibody (Ventana Medical Systems) at the National Cancer Center Hospital East. The intensities of membrane staining for EGFR and c-MET were scored as described for HER2. We defined HER2, EGFR, and c-MET positivity as an IHC membrane staining intensity score of $2+$ or $3+$.

\section{Case selection and methods for HER2, EGFR, and C-MET IHC}

Based on the results of IHC in TMA, cases were selected for detailed examination. The following conditions were employed for case selection: (1) predominant IHC score was $3+$, but concomitant with 2+ or less, (2) co-overexpression cases, and (3) existence of mucosal lesions. For these 
selection cases, HER2, EGFR, and c-MET expression levels were investigated in more than three tumor blocks per case using IHC. We re-evaluated IHC score and comparatively evaluated differences in IHC expression status in each molecule between the mucosal portion (MuP) and invasive portion (InP) based on histological findings. We defined MuP as submucosal or deeper invasion with invasive front and InP as mucosal lesion without submucosal or deeper invasion.

For assessment of the difference in HER2, EGFR, and c-MET expression statuses, the IHC scores in the MuP and InP were independently evaluated, and the results were divided into four patterns: $\mathrm{MuP}=\mathrm{InP}$ (Fig. 1a), MuP $>\operatorname{InP}$ (Fig. 1b), MuP < InP (Fig. 1c), and IHC negative, but partly high expression (Fig. 1d). For RTK-positive cases, $\mathrm{MuP}=\mathrm{InP}$ was defined as equivalent IHC scores in the MuP and InP. MuP $>$ InP was defined as having a higher IHC score in the MuP than in the InP. MuP $<$ InP was defined as having a lower IHC score in the MuP than in the InP.

\section{Statistical analysis}

The baseline characteristics of each case were compared using $\chi^{2}$ tests or Fisher's exact tests for categorical data. Mann-Whitney tests were used for non-normally distributed continuous variables. Differences with $p$ values of less than 0.05 (two-sided) were considered statistically significant. All

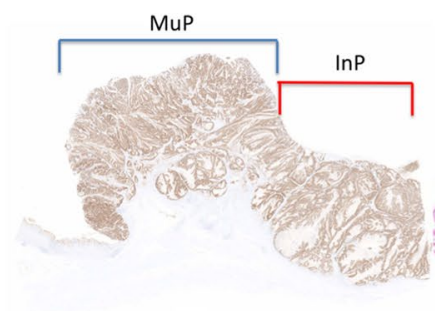

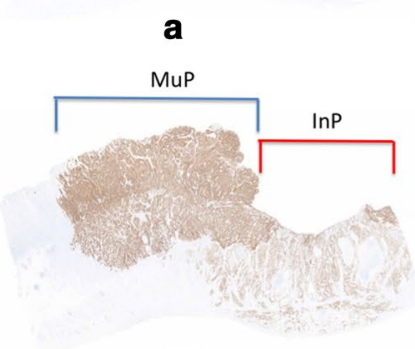

c

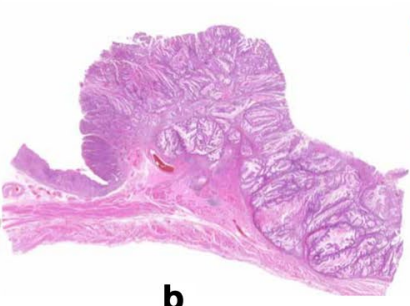

b

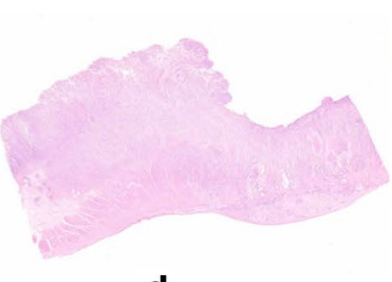

d
Fig. 1 Assessment of differences in IHC expression statuses. a Mucosal portion =invasive portion. In this case, HER2 status was positive in both the mucosal and invasive portions. The blue line shows the mucosal portion, while the red line shows the invasive portion. b Histologically, the resected specimen revealed a papillary adenocarcinoma deeply extending into the subserosa. c Mucosal portion > invasive portion. In this case, HER2 status was positive in the mucosal portion and negative in the invasive portion. The blue line shows the mucosal portion, while the red line shows the invasive portion. d Histologically, the resected specimen revealed a moderately analyses were performed using SPSS version 22.0 (SPSS Inc., Chicago, IL, USA).

\section{Results}

\section{HER2, EGFR, and c-MET levels}

In this study, a total of 375 patients fulfilled all study criteria and were included for evaluation of HER2, EGFR, and c-MET statuses using IHC. The baseline characteristics of these patients are shown in Table 1. The HER2 IHC score was 0 or $1+$ in 353 cases (94.1\%), 2+ in eight cases (2.1\%), and $3+$ in 14 cases (3.7\%). The EGFR IHC score was 0 or $1+$ in 343 cases $(91.5 \%), 2+$ in 29 cases $(7.7 \%)$, and $3+$ in three cases $(0.8 \%)$. The c-MET IHC score was 0 or $1+$ in 299 cases $(79.7 \%), 2+$ in 71 cases $(18.9 \%)$, and $3+$ in five cases (1.3\%). In total, the HER2-, EGFR-, and c-METpositive rates were $5.9 \%(22 / 375), 8.5 \%$ (32/375), and $20.3 \%$ (76/375), respectively (Table 2 ).

\section{HER2, EGFR, and c-MET expression statuses and clinicopathological parameters}

The correlations between HER2, EGFR, and c-MET expression statuses and clinicopathological parameters are shown in Table 3. There were no significant differences in

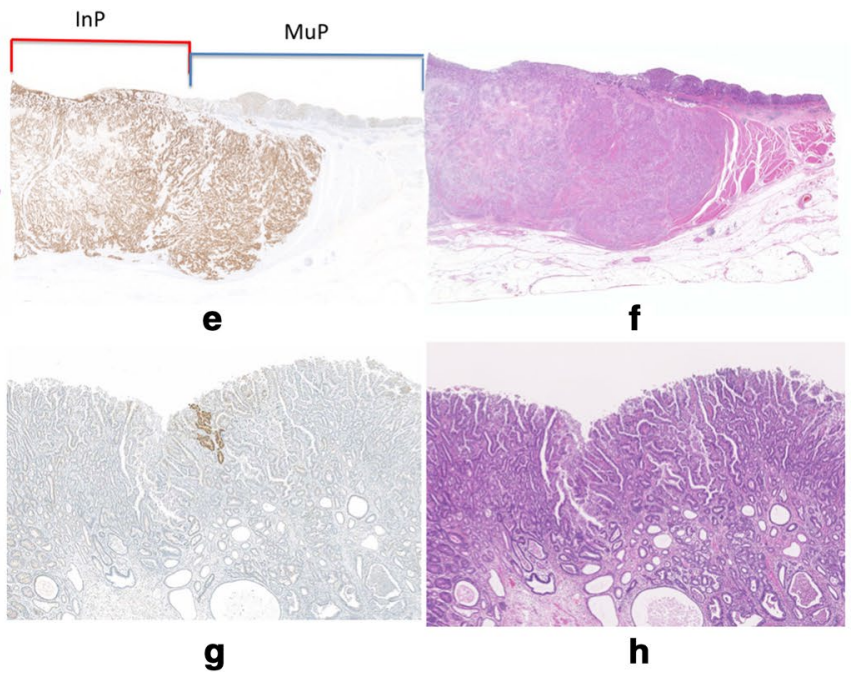

differentiated adenocarcinoma deeply extending into the subserosa. e Mucosal portion <invasive portion. In this case, c-MET status was positive in the invasive portion and negative in the mucosal portion. The blue line shows the mucosal portion, while the red line shows the invasive portion. f Histologically, the resected specimen revealed a moderately differentiated adenocarcinoma deeply extending into the subserosa. d IHC negative, but partial high expression. In this case, EGFR status was negative, but partial high expression was present in the mucosa. h Histologically, the resected specimen revealed a papillary adenocarcinoma extending into the submucosa 
Table 1 Baseline characteristics

\begin{tabular}{ll}
\hline & Total, $n(\%)$ \\
\hline Enrolled patients, $n$ & 375 \\
Gender, $n(\%)$ & \\
Male & $269(72)$ \\
Female & $106(28)$ \\
Age, years & \\
Median (range) & $67(31-93)$ \\
Primary tumor location, $n(\%)$ & \\
Gastro-esophageal junction & $26(7)$ \\
Stomach & $349(93)$ \\
Histological classification, $n(\%)$ & \\
Intestinal type & $210(56)$ \\
Diffuse type & $165(44)$ \\
Borrmann classification, $n(\%)$ & \\
0 & $130(35)$ \\
1 & $10(3)$ \\
2 & $86(23)$ \\
3 & $122(33)$ \\
4 & $27(7)$ \\
T stage (AJCC 7th edition) & \\
0 & $3(1)$ \\
1 & $75(20)$ \\
3 & $78(21)$ \\
4 & $92(24)$ \\
\hline
\end{tabular}

sex, age, primary tumor location, Borrmann classification, and $\mathrm{T}$ stage with relation to HER2, EGFR, and c-MET expression statuses.

The proportion of HER2-positive GCs with an intestinal type was significantly higher than that of tumors with a diffuse type (9.0 versus $1.8 \%$, respectively; $p=0.003$ ). Similarly, the proportions of EGFR- and c-MET-positive tumors with an intestinal type were significantly higher than those with a diffuse type (EGFR: 11.9 versus $4.2 \%$, respectively, $p=0.009$; c-MET: 26.7 versus $12.1 \%$, respectively, $p<0.001)$. Among intestinal-type tumors, the HER2-positive rate of papillary adenocarcinomas (versus tubular adenocarcinomas) was particularly high $(25.8 \%$, $8 / 31 ; p=0.002$ ).

\section{Case selection and differences in IHC expression statuses}

To assess differences in IHC results in the same lesion, we selected 22 cases (12 HER2-positive cases, 11 EGFR-positive cases, and $12 \mathrm{c}$-MET-positive cases). In the selected cases, co-overexpression of HER2, EGFR, and/or c-MET was observed for 10 cases, including four cases of triplepositive cancer, one case of HER2- and EGFR-positive cancer, three cases of HER2- and c-MET-positive cancer, and two cases of EGFR- and c-MET-positive cancer. After re-evaluation of more than three tumor blocks per case, 13 cases were HER2-positive, five cases were EGFR-positive, nine cases were c-MET-positive, and four cases were IHC negative.

Table 4 shows differences in IHC expression statuses. In HER2-positive GCs, the rates of $\mathrm{MuP}=\mathrm{InP}, \mathrm{MuP}>\mathrm{InP}$, and $\mathrm{MuP}<\mathrm{InP}$ were 46, 54, and 0\%, respectively. In EGFR-positive $\mathrm{GCs}$, the rates of $\mathrm{MuP}=\mathrm{InP}, \mathrm{MuP}>\mathrm{InP}$, and $\mathrm{MuP}<\mathrm{InP}$ were 40,0 , and $60 \%$, respectively. In EGFR-negative GCs, three cases (14.3\%) showed partly high expression of EGFR. In c-MET-positive GCs, the rates of $\mathrm{MuP}=\mathrm{InP}, \mathrm{MuP}>\mathrm{InP}$, and $\mathrm{MuP}<\mathrm{InP}$ were 11,44 , and $44 \%$, respectively. In EGFRnegative GCs, three cases $(17.6 \%)$ showed partial high expression of EGFR.

Based on these data, we calculated the concordance rate of IHC expression status in the MuP or InP compared with the whole tumor (Table 5). Concordance rates of positive staining of HER2, EGFR, and MET between MuP and whole tumor were 100, 40, and 56\%, and those with InP were 46, 100, and 56\%, respectively. In HER2-positive GCs, HER2 was more frequently positive in the MuP of the tumor than in the InP ( $p=0.003)$. In EGFR-positive GCs, there was no significant difference between the MuP and InP, although EGFR tended to be positive more frequently in the InP than in the MuP $(p=0.167)$. In c-MET-positive GCs, the frequency of c-MET expression did not differ between the MuP and $\operatorname{InP}(p=1.000)$.

Based on endoscopic findings, we calculated the concordance rate of IHC expression status in the InP with or without ulceration. In HER2-positive GCs, the concordance rate in the InP with ulceration was 56\% (5/9; Fig. 2a, b), whereas that without ulceration was 25\% (1/4; Fig. 2c, d). In EGFRpositive GCs, sample size was small, hence the differences
Table 2 Human epidermal growth factor 2, epidermal growth factor receptor, and mesenchymal epithelial transition factor data

\begin{tabular}{lccc}
\hline & HER2 & EGFR & c-MET \\
\hline IHC score $0,1+(n, \%)$ & $353(94.1 \%)$ & $343(91.5 \%)$ & $299(79.7 \%)$ \\
IHC score $2+(n, \%)$ & $8(2.1 \%)$ & $29(7.7 \%)$ & $71(18.9 \%)$ \\
IHC score $3+(n, \%)$ & $14(3.7 \%)$ & $3(0.8 \%)$ & $5(1.3 \%)$ \\
Positive $(2+$ or $3+)(n, \%)$ & $22 / 375(5.9 \%)$ & $32 / 375(8.5 \%)$ & $76 / 375(20.3 \%)$ \\
\hline
\end{tabular}


Table 3 Correlations between human epidermal growth factor 2, epidermal growth factor receptor, and mesenchymal epithelial transition factor expression statuses and clinicopathological parameters

\begin{tabular}{|c|c|c|c|c|c|c|c|}
\hline & \multirow[t]{2}{*}{ Total } & \multicolumn{2}{|c|}{ HER2 status } & \multicolumn{2}{|c|}{ EGFR status } & \multicolumn{2}{|c|}{ c-MET status } \\
\hline & & Positive & Positive rate $(\%)$ & Positive & Positive rate $(\%)$ & Positive & Positive rate $(\%)$ \\
\hline Enrolled patients, $n$ & 375 & 22 & 5.9 & 32 & 8.5 & 76 & 20.3 \\
\hline Gender, $n(\%)$ & & & $P=1.000$ & & $P=1.000$ & & $P=0.776$ \\
\hline Male & $269(72)$ & $16(73)$ & 5.9 & $23(72)$ & 8.6 & $56(74)$ & 20.8 \\
\hline Female & $106(28)$ & $6(27)$ & 5.7 & $9(28)$ & 8.5 & $20(26)$ & 18.9 \\
\hline Age, years & & & $P=0.820$ & & $P=1.000$ & & $P=0.285$ \\
\hline$<65$ & $135(36)$ & $7(32)$ & 5.2 & $11(34)$ & 8.1 & $23(30)$ & 17.0 \\
\hline$\geq 65$ & $240(64)$ & $15(68)$ & 6.3 & $21(66)$ & 8.8 & $53(70)$ & 22.1 \\
\hline Primary tumor location, $n(\%)$ & & & $P=0.189$ & & $P=0.261$ & & $P=0.800$ \\
\hline Gastro-esophageal junction & $26(7)$ & $3(14)$ & 11.5 & $4(13)$ & 15.4 & $6(8)$ & 23.1 \\
\hline Stomach & $349(93)$ & $19(86)$ & 5.4 & $28(87)$ & 8.0 & $70(92)$ & 20.1 \\
\hline Histological classification, $n(\%)$ & & & $P=0.003$ & & $P=0.009$ & & $P<0.001$ \\
\hline Diffuse type & $165(44)$ & $3(14)$ & 1.8 & $7(22)$ & 4.2 & $20(26)$ & 12.1 \\
\hline Intestinal type & $210(56)$ & $19(86)$ & 9.0 & $25(78)$ & 11.9 & $56(74)$ & 26.7 \\
\hline Tubular $(1,2)$ adenocarcinoma & $179(85)$ & $11(58)$ & $6.1 P=0.002$ & $18(72)$ & $10.1 P=0.067$ & $48(86)$ & $26.8 P=1.000$ \\
\hline Papillary adenocarcinoma & $31(15)$ & $8(42)$ & 25.8 & $7(28)$ & 22.6 & $8(14)$ & 25.8 \\
\hline Borrmann classification, $n(\%)$ & & & $P=0.370$ & & $P=0.180$ & & $P=0.234$ \\
\hline 0 & $130(35)$ & $4(18)$ & 3.1 & $7(22)$ & 5.4 & $31(41)$ & 23.8 \\
\hline 1 & $10(3)$ & $2(9)$ & 20.0 & $1(3)$ & 10.0 & $2(3)$ & 20.0 \\
\hline 2 & $86(23)$ & $11(50)$ & 12.8 & $9(28)$ & 10.5 & $18(23)$ & 20.9 \\
\hline 3 & $122(33)$ & $5(23)$ & 4.1 & $15(47)$ & 12.3 & $23(30)$ & 18.9 \\
\hline 4 & $27(7)$ & $0(0)$ & 0 & $0(0)$ & 0 & $2(3)$ & 7.4 \\
\hline T stage (AJCC 7th edition) & & & $P=0.588$ & & $P=0.648$ & & $P=0.114$ \\
\hline 0 & $3(1)$ & $1(5)$ & 33.3 & $0(0)$ & 0 & $2(3)$ & 66.7 \\
\hline 1 & $75(20)$ & $2(9)$ & 2.7 & $5(16)$ & 6.7 & $19(25)$ & 25.3 \\
\hline 2 & $78(21)$ & $4(18)$ & 5.1 & $6(19)$ & 7.7 & $17(22)$ & 21.8 \\
\hline 3 & $92(24)$ & $7(32)$ & 7.6 & $10(31)$ & 10.9 & $16(21)$ & 17.4 \\
\hline 4 & $127(34)$ & $8(36)$ & 6.3 & $11(34)$ & 8.7 & $22(29)$ & 17.3 \\
\hline
\end{tabular}

Table 4 Differences in IHC expression statuses of the mucosal and invasive portions

\begin{tabular}{llllr}
\hline & $\begin{array}{l}\text { Mucosal por- } \\
\text { tion=invasive portion }\end{array}$ & $\begin{array}{l}\text { Mucosal por- } \\
\text { tion> invasive portion }\end{array}$ & $\begin{array}{l}\text { Mucosal por- } \\
\text { tion<invasive portion }\end{array}$ & $\begin{array}{l}\text { IHC posi- } \\
\text { tive case } \\
(n)\end{array}$ \\
\hline HER2 $(n, \%)$ & $6(46 \%)$ & $7(54 \%)$ & $0(0 \%)$ & 13 \\
EGFR $(n, \%)$ & $2(40 \%)$ & $0(0 \%)$ & $3(60 \%)$ & 5 \\
c-MET $(n, \%)$ & $1(11 \%)$ & $4(44 \%)$ & $4(44 \%)$ & 9 \\
\hline
\end{tabular}

Table 5 Concordance of positive staining of HER2, EGFR and MET between $\mathrm{MuP}$ and whole tumor, and those with $\mathrm{InP}$ and whole tumor

\begin{tabular}{lccc}
\hline & Mucosal portion & Invasive portion & $p$ value \\
\hline HER2 $(\%)$ & $13 / 13(100 \%)$ & $6 / 13(46 \%)$ & 0.003 \\
EGFR $(\%)$ & $2 / 5(40 \%)$ & $5 / 5(100 \%)$ & 0.167 \\
c-MET $(\%)$ & $5 / 9(56 \%)$ & $5 / 9(56 \%)$ & 1.000 \\
\hline
\end{tabular}

were unclear. In c-MET-positive GCs, c-MET expression showed no clear differences in staining. 
Fig. 2 a Endoscopic examination showed a type 2 tumor in the greater curvature of the gastric upper body of the stomach. In this tumor, there was an invasive portion with ulceration. b Histologically, HER2 status was positive in the mucosal portion and negative in invasive portion. c Endoscopic examination showed a type 1 tumor in the posterior wall of the gastric lower body of the stomach. In this tumor, there was an invasive portion without ulceration. d Histologically, HER2 status was positive in the mucosal portion and negative in the invasive portion including the surface mucosa

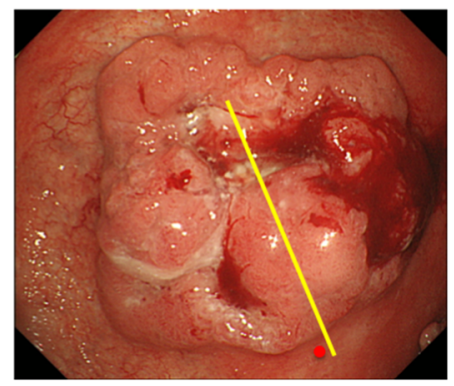

a

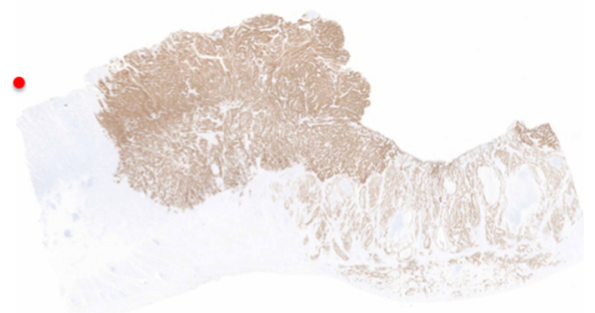

b

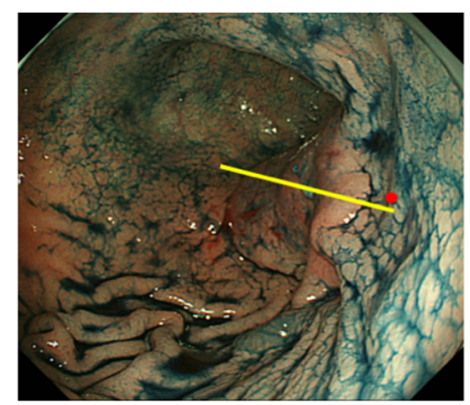

C

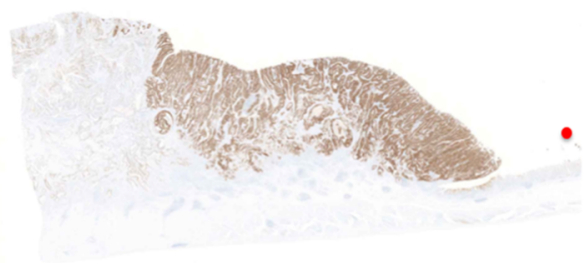

\section{Discussion}

Previous reports have shown the correlations between HER2, EGFR, and c-MET expression status and clinicopathological parameters in GC. HER2-positive GCs are more frequently of the intestinal type (papillary and tubular adenocarcinoma), associated with older age, and predominantly found in men compared with HER2-negative GCs. Additionally, EGFR-positive GCs more frequently show a moderately to poorly differentiated histopathology, are associated with older age, and are observed with advanced tumor stage compared with EGFR-negative GCs [12, 13]. Finally, c-MET-positive GCs are more frequently of the intestinal type and associated with an advanced clinical stage compared with c-MET-negative GCs [17, 19, 32]. In contrast, in our study, there were no significant differences in sex, age, primary tumor location, Borrmann classification, and T stage based on HER2, EGFR, and c-MET expression statuses. The proportions of HER2-, EGFR-, and c-METpositive GCs with an intestinal type were significantly higher than those of GCs with diffuse type. Among intestinal-type tumors, the HER2-positive rate of papillary adenocarcinomas (versus tubular adenocarcinomas) was particularly high. The biological reasons for these associations between HER2, EGFR, and c-MET overexpression and the histological type of GC have not been fully elucidated, and additional investigations are needed.

In this study, based on the results of IHC in TMA, cases were selected for detailed examination. TMA is a very useful tool for analyzing large numbers of cases; however, the results often exhibit sampling bias. One of the reasons for the discrepancy between TMA and whole tissue sections is thought to be the heterogeneity of RTK overexpression [25]. In this study, the frequency of HER2 overexpression was lower than that in a previous report [11]. Regarding the evaluation method of HER2 overexpression, we used a $10 \%$ cut-off value that was established for surgical sample evaluation. However, only small amounts of tissue were used in TMAs. Therefore, a better estimate may be obtained in a single cluster of at least five positive cells, which is sufficient for endoscopic biopsy samples. Moreover, in this study, TMAs were obtained from the invasive front. Therefore, the HER2 overexpression rate may be an underestimation. Although the IHC staining status is heterogeneous in GC, the TMA method can provide meaningful information when used in a large cohort.

Indeed, RTKs have been shown to be heterogeneously expressed in GC. Previous reports have shown that samples with strong staining for HER2 were homogeneously stained, whereas samples with strong staining for EGFR and c-MET exhibited highly heterogeneous staining [25, 27, 28]. Clinically, RTKs are usually evaluated from only a small fraction of the primary tumor, usually derived from a biopsy. Therefore, intratumor heterogeneity may be a potential cause for the failure of targeted therapy in GC.

PIT is a new class of molecular-targeted cancer therapy that combines the specificity of antibodies for targeting tumors with the toxicity induced by photosensitizers after exposure to near infrared light. PIT shows highly specific cytotoxicity in tumor cells expressing particular antigens [33]. The choice of monoclonal antibody in photosensitizer conjugates may influence the effectiveness of PIT. Recent studies have shown effective tumor control, including of HER2- or EGFR-positive cancer, by selective 
molecular targeting with PIT [34-39]. Moreover, a previous report found that multi-epitope targeting can significantly enhance the efficacy of HER2-targeted PIT, and more generally, multi-epitope targeting should be advantageous for PIT of other cell-surface receptors that are frequently overexpressed in various cancers (e.g., EGFR, MET, and fibroblast growth factor receptors) [40]. Therefore, in the future, it may become important to recognize the RTK-positive site by selective molecular targeting.

Previous studies have reported that the mucosal and invasive fronts of tumors showed no preference for HER2 staining [25], whereas other studies have reported that HER2 expression was preferentially observed in the luminal lateral layer of the tumor and not in the deeper central layer [41, 42]. We previously reported that HER2 was more frequently positive in the superficial spreading portion than in the ulcer bed of the tumors using biopsy samples [43]. Similarly, in this study, we found that HER2 was more frequently positive in the MuP of the tumor than in the InP using surgical samples, potentially because of the poorer differentiation of deeper layers in GCs. Indeed, a previous report showed that poorly differentiated tumors tended to have high rates of heterogeneity for HER2 expression [44]. Based on endoscopic findings, HER2 was frequently negative in the InP, with or without ulceration, in our study. Therefore, to avoid underestimation of HER2 expression status, biopsies must be taken from the tumor edge without invasion.

Notably, samples with strong staining for EGFR have been shown to exhibit highly heterogeneous staining; however, no reports have further characterized the features of these tumors [28]. In this study, we found that EGFR expression in the InP was higher than that in the MuP, and EGFR expression was highly heterogeneous. Although the clinical significance is unknown, in EGFR-negative GCs, three cases (17.6\%) showed partial high expression of EGFR.

A previous study reported that heterogeneous expression of c-MET was common in GC; therefore, comprehensive evaluation of c-MET status in different sites within the tumor was recommended [27]. Similarly, in this study, we found that c-MET expression did not differ between the MuP and InP.

In this study, we evaluated HER2, EGFR, and c-MET status based on surgical samples. HER2 was more frequently positive in the MuP of the tumor than in the InP. Moreover, regarding the evaluation method of HER 2 overexpression, the use of a single cluster of at least five positive cells from endoscopic biopsy samples is preferred, as the possibility that HER2 expression will be underestimated is low for endoscopic biopsy samples. As for EGFR and c-MET, several cases exhibited the pattern $\mathrm{MuP}<\mathrm{InP}$. Therefore, the possibility that EGFR and c-MET expression will be underestimated is high for endoscopic biopsy samples.
Our study had several limitations. First, the diagnostic criteria for EGFR and c-MET status were tentative and have not been standardized. Second, we analyzed HER2, EGFR, and c-MET expression only at the protein level using IHC, and we did not examine gene amplification. Thus, further studies are required to confirm and expand upon our findings.

\section{Conclusions}

In this study, we investigated the differences in heterogeneity of HER2, EGFR, and c-MET expression based on the macroscopic appearance of GC. To avoid underestimation of expression status, biopsies must be taken from the MuP (particularly the tumor edge without invasion) for HER2, the InP for EGFR, and the MuP and InP for c-MET.

\section{Compliance with ethical standards}

Conflict of interest Yasuhiro Oono, Takeshi Kuwata, Kenji Takashima, Kensuke Shinmura, Keisuke Hori, Yusuke Yoda, Hiroaki Ikematsu, and Tomonori Yano have no conflict of interests or financial ties to disclose. Kohei Shitara reports personal fees from Astellas Pharma, grants and personal fees from Lilly, personal fees from BristolMyers Squibb, personal fees from Takeda, personal fees from Pfizer, grants and personal fees from Ono Pharmaceutical, personal fees from Novartis, personal fees from Abbvie, personal fees from Yakult, grants from Dainippon Sumitomo Pharma, grants from MSD, grants from Daiichi Sankyo, grants from Taiho Pharmaceutical, and grants from Chugai Pharma, outside the submitted work. Takahiro Kinoshita reports personal fees from Olympus, Johnson \& Johnson, Medtronic, Yakult Pharma, Taiho Pharma, and Eli Lilly, outside the submitted work.

Ethical considerations The study protocol was approved by the medical ethics committee of our hospital (2013-336). Patient informed consent was waived due to the retrospective design of the study. This study was performed in accordance with the ethical principles outlined in the Declaration of Helsinki.

\section{References}

1. Global Burden of Disease Cancer C, Fitzmaurice C, Allen C, Barber RM, Barregard L, Bhutta ZA, et al. Global, regional, and national cancer incidence, mortality, years of life lost, years lived with disability, and disability-adjusted life-years for 32 cancer groups, 1990 to 2015: a systematic analysis for the global burden of disease study. JAMA Oncol. 2017;3:524-48.

2. Landry J, Tepper JE, Wood WC, Moulton EO, Koerner F, Sullinger J. Patterns of failure following curative resection of gastric carcinoma. Int J Radiat Oncol Biol Phys. 1990;19:1357-62.

3. Yoo CH, Noh SH, Shin DW, Choi SH, Min JS. Recurrence following curative resection for gastric carcinoma. Br J Surg. 2000;87:236-42.

4. Lim DH, Kim DY, Kang MK, Kim YI, Kang WK, Park $\mathrm{CK}$, et al. Patterns of failure in gastric carcinoma after D2 
gastrectomy and chemoradiotherapy: a radiation oncologist's view. Br J Cancer. 2004;91:11-7.

5. Cunningham D, Humblet Y, Siena S, Khayat D, Bleiberg H, Santoro A, et al. Cetuximab monotherapy and cetuximab plus irinotecan in irinotecan-refractory metastatic colorectal cancer. N Engl J Med. 2004;351:337-45.

6. Luis M, Tavares A, Carvalho LS, Lara-Santos L, Araujo A, de Mello RA. Personalizing therapies for gastric cancer: molecular mechanisms and novel targeted therapies. World J Gastroenterol. 2013;19:6383-97.

7. Scagliotti GV, Novello S, von Pawel J. The emerging role of MET/HGF inhibitors in oncology. Cancer Treat Rev. 2013;39:793-801.

8. Bang YJ, Van Cutsem E, Feyereislova A, Chung HC, Shen L, Sawaki A, et al. Trastuzumab in combination with chemotherapy versus chemotherapy alone for treatment of HER2-positive advanced gastric or gastro-oesophageal junction cancer (ToGA): a phase 3, open-label, randomised controlled trial. Lancet. 2010;376:687-97.

9. Soularue E, Cohen R, Tournigand C, Zaanan A, Louvet C, Bachet $\mathrm{JB}$, et al. Efficacy and safety of trastuzumab in combination with oxaliplatin and fluorouracil-based chemotherapy for patients with HER2-positive metastatic gastric and gastro-oesophageal junction adenocarcinoma patients: a retrospective study. Bull Cancer. 2015;102:324-31.

10. Gravalos C, Gomez-Martin C, Rivera F, Ales I, Queralt B, Marquez A, et al. Phase II study of trastuzumab and cisplatin as first-line therapy in patients with HER2-positive advanced gastric or gastroesophageal junction cancer. Clin Transl Oncol. 2011;13:179-84.

11. Jorgensen JT, Hersom M. HER2 as a prognostic marker in gastric cancer - a systematic analysis of data from the literature. J Cancer. 2012;3:137-44.

12. Galizia G, Lieto E, Orditura M, Castellano P, Mura AL, Imperatore V, et al. Epidermal growth factor receptor (EGFR) expression is associated with a worse prognosis in gastric cancer patients undergoing curative surgery. World J Surg. 2007;31:1458-68.

13. Kim MA, Lee HS, Lee HE, Jeon YK, Yang HK, Kim WH. EGFR in gastric carcinomas: prognostic significance of protein overexpression and high gene copy number. Histopathology. 2008;52:738-46.

14. Hayashi M, Inokuchi M, Takagi Y, Yamada H, Kojima K, Kumagai J, et al. High expression of HER3 is associated with a decreased survival in gastric cancer. Clin Cancer Res. 2008;14:7843-9.

15. Terashima M, Kitada K, Ochiai A, Ichikawa W, Kurahashi I, Sakuramoto S, et al. Impact of expression of human epidermal growth factor receptors EGFR and ERBB2 on survival in stage II/III gastric cancer. Clin Cancer Res. 2012;18:5992-6000.

16. Tsugawa K, Yonemura Y, Hirono Y, Fushida S, Kaji M, Miwa K, et al. Amplification of the c-met, c-erbB-2 and epidermal growth factor receptor gene in human gastric cancers: correlation to clinical features. Oncology. 1998;55:475-81.

17. Huang TJ, Wang JY, Lin SR, Lian ST, Hsieh JS. Overexpression of the c-met protooncogene in human gastric carcinoma-correlation to clinical features. Acta Oncol. 2001;40:638-43.

18. Lee HE, Kim MA, Lee HS, Jung EJ, Yang HK, Lee BL, et al. MET in gastric carcinomas: comparison between protein expression and gene copy number and impact on clinical outcome. Br J Cancer. 2012;107:325-33.

19. Ha SY, Lee J, Kang SY, Do IG, Ahn S, Park JO, et al. MET overexpression assessed by new interpretation method predicts gene amplification and poor survival in advanced gastric carcinomas. Mod Pathol. 2013;26:1632-41.

20. An X, Wang F, Shao Q, Wang FH, Wang ZQ, Wang ZQ, et al. MET amplification is not rare and predicts unfavorable clinical outcomes in patients with recurrent/metastatic gastric cancer after chemotherapy. Cancer. 2014;120:675-82.

21. Fuse N, Kuboki Y, Kuwata T, Nishina T, Kadowaki S, Shinozaki E, et al. Prognostic impact of HER2, EGFR, and c-MET status on overall survival of advanced gastric cancer patients. Gastric Cancer. 2016;19:183-91.

22. Kim MA, Lee HJ, Yang HK, Bang YJ, Kim WH. Heterogeneous amplification of ERBB2 in primary lesions is responsible for the discordant ERBB2 status of primary and metastatic lesions in gastric carcinoma. Histopathology. 2011;59:822-31.

23. Grabsch H, Sivakumar S, Gray S, Gabbert HE, Muller W. HER2 expression in gastric cancer: rare, heterogeneous and of no prognostic value-conclusions from 924 cases of two independent series. Cell Oncol. 2010;32:57-65.

24. Grillo F, Fassan M, Ceccaroli C, Giacometti C, Curto M, Zagonel $\mathrm{V}$, et al. The reliability of endoscopic biopsies in assessing her2 status in gastric and gastroesophageal junction cancer: a study comparing biopsies with surgical samples. Transl Oncol. 2013;6:10-6.

25. Kim KC, Koh YW, Chang HM, Kim TH, Yook JH, Kim BS, et al. Evaluation of HER2 protein expression in gastric carcinomas: comparative analysis of 1414 cases of whole-tissue sections and 595 cases of tissue microarrays. Ann Surg Oncol. 2011;18:2833-40.

26. Hofmann M, Stoss O, Shi D, Buttner R, van de Vijver M, Kim W, et al. Assessment of a HER2 scoring system for gastric cancer: results from a validation study. Histopathology. 2008;52:797-805.

27. Zhu C, Xu J, Li M, Zhao G, Cao H. Heterogeneity of c-Met expression in Chinese gastric cancer patients. Hum Pathol. 2015;46:1901-7.

28. Nagatsuma AK, Aizawa M, Kuwata T, Doi T, Ohtsu A, Fujii H, et al. Expression profiles of HER2, EGFR, MET and FGFR2 in a large cohort of patients with gastric adenocarcinoma. Gastric Cancer. 2015;18:227-38.

29. Lordick F, Shitara K, Janjigian YY. New agents on the horizon in gastric cancer. Ann Oncol. 2017;28:1767-1775

30. Aizawa M, Nagatsuma AK, Kitada K, Kuwata T, Fujii S, Kinoshita T, et al. Evaluation of HER2-based biology in 1006 cases of gastric cancer in a Japanese population. Gastric Cancer. 2014; 17:34-42.

31. Siewert JR, Stein HJ. Classification of adenocarcinoma of the oesophagogastric junction. Br J Surg. 1998;85:1457-9.

32. Sotoudeh K, Hashemi F, Madjd Z, Sadeghipour A, Molanaei S, Kalantary E. The clinicopathologic association of c-MET overexpression in Iranian gastric carcinomas; an immunohistochemical study of tissue microarrays. Diagn Pathol. 2012;7:57.

33. Mitsunaga M, Ogawa M, Kosaka N, Rosenblum LT, Choyke PL, Kobayashi H. Cancer cell-selective in vivo near infrared photoimmunotherapy targeting specific membrane molecules. Nat Med. 2011;17:1685-91.

34. Shimoyama K, Kagawa S, Ishida M, Watanabe S, Noma K, Takehara K, et al. Viral transduction of the HER2-extracellular domain expands trastuzumab-based photoimmunotherapy for HER2-negative breast cancer cells. Breast Cancer Res Treat. 2015;149:597-605.

35. Sato K, Choyke PL, Kobayashi H. Photoimmunotherapy of gastric cancer peritoneal carcinomatosis in a mouse model. PLoS One. 2014;9:e113276.

36. Sato K, Nagaya T, Choyke PL, Kobayashi H. Near infrared photoimmunotherapy in the treatment of pleural disseminated NSCLC: preclinical experience. Theranostics. 2015;5:698-709.

37. Ito K, Mitsunaga M, Nishimura T, Kobayashi H, Tajiri H. Combination photoimmunotherapy with monoclonal antibodies recognizing different epitopes of human epidermal growth factor receptor 2: an assessment of phototherapeutic effect based on fluorescence molecular imaging. Oncotarget. 2016;7:14143-52. 
38. Sato K, Watanabe R, Hanaoka H, Harada T, Nakajima T, Kim I, et al. Photoimmunotherapy: comparative effectiveness of two monoclonal antibodies targeting the epidermal growth factor receptor. Mol Oncol. 2014;8:620-32.

39. Ito K, Mitsunaga M, Nishimura T, Saruta M, Iwamoto T, Kobayashi $\mathrm{H}$, et al. Near-infrared photochemoimmunotherapy by photoactivatable bifunctional antibody-drug conjugates targeting human epidermal growth factor receptor 2 positive cancer. Bioconj Chem. 2017;28:1458-69.

40. Savellano MD, Pogue BW, Hoopes PJ, Vitetta ES, Paulsen KD. Multiepitope HER2 targeting enhances photoimmunotherapy of HER2-overexpressing cancer cells with pyropheophorbide-a immunoconjugates. Cancer Res. 2005;65:6371-9.

41. Lee S, de Boer WB, Fermoyle S, Platten M, Kumarasinghe MP. Human epidermal growth factor receptor 2 testing in gastric carcinoma: issues related to heterogeneity in biopsies and resections. Histopathology. 2011;59:832-40.

42. Tominaga N, Gotoda T, Hara M, Hale MD, Tsuchiya T, Matsubayashi $\mathrm{J}$, et al. Five biopsy specimens from the proximal part of the tumor reliably determine HER 2 protein expression status in gastric cancer. Gastric Cancer. 2016;19:553-60.

43. Oono Y, Kuwata T, Takashima K, Yoda Y, Ikematsu H, Shitara $\mathrm{K}$, et al. Clinicopathological features and endoscopic findings of HER2-positive gastric cancer. Surg Endosc. 2018. https://doi. org/10.1007/s00464-018-6138-8

44. Nishida Y, Kuwata T, Nitta H, Dennis E, Aizawa M, Kinoshita $\mathrm{T}$, et al. A novel gene-protein assay for evaluating HER2 status in gastric cancer: simultaneous analyses of HER2 protein overexpression and gene amplification reveal intratumoral heterogeneity. Gastric Cancer. 2015;18:458-66. 